\title{
Phases changes of the AK12MMgN-18\%SiCp composite alloy after severe plastic deformation and annealing
}

\author{
G.R. Khalikova ${ }^{\dagger}$, G.F. Korznikova, V.G. Trifonov \\ †gulnara.r.khalikova@gmail.com
}

Institute for Metals Superplasticity Problems RAS, 39 Khalturin St., Ufa, 450001, Russia

The effect of severe plastic deformation by high pressure torsion (HPT) on the structure of AK12MMgN-18\%SiCp composite alloy was investigated. A liquid-forged billet was taken as an initial material. Samples $8 \mathrm{~mm}$ in diameter and $0.45 \mathrm{~mm}$ in thickness were deformed by torsion in a Bridgman anvil at room temperature up to 5 turns under the pressure of $4 \mathrm{GPa}$. After deformation the samples were annealed in the temperature range of $300-500^{\circ} \mathrm{C}$ for 5 minutes. The average area and volume fraction of particles in the initial, HPT-processed and annealed samples were evaluated. The change of the concentration level of a solid solution in the states under study was analyzed. It was shown that HPT led to a fragmentation of particles and a reduction of their average area: silicon particles from $4.4 \pm 0.1$ to $0.32 \pm 0.02 \mu \mathrm{m}^{2}$, intermetallic particles from $5.2 \pm 2.6$ to $0.20 \pm 0.02 \mu \mathrm{m}^{2}$, and $\mathrm{SiC}$ from $37.6 \pm 0.9$ to $3.9 \pm 0.02 \mu \mathrm{m}^{2}$. At the same time, a partial dissolution of excess phases occurred that resulted in the formation of a supersaturated solid solution. Post-HPT annealing led to a dissolution of the supersaturated solid solution with a separation of secondary phases of different morphology not typical to the alloy: globular silicon, rod shaped phase enriched by $\mathrm{Ni}$, Si and Fe and a more compact phase containing $\mathrm{Si}$ and $\mathrm{Cu}$. The higher the annealing temperature, the more intensive was the process of supersaturated solid solution decomposition and the higher was the volume fraction of silicon and intermetallic particles. Their average area increased with annealing temperature. No changes in the average area and volume fraction of $\mathrm{SiC}$ particles during annealings of HPT-processed alloy were observed.

Keywords: HPT, aluminum, solid solution, dissociation.

\section{Фазовые изменения в композиционном сплаве AК12ММrH-18\%SiCp после интенсивной пластической деформации и отжига}

\author{
Халикова Г.Р. ${ }^{\dagger}$, Корзникова Г.Ф., Трифонов В.Г. \\ †gulnara.r.khalikova@gmail.com
}

Институт проблем сверхпластичности металлов РАН, ул. Халтурина 39, 450001, Уфа, Россия

Исследовано влияние интенсивной пластической деформации, реализованное кручением под высоким давлением на структуру композиционного сплава АК12MМгH-18\%SiCp. Исходным материалом служила жидкоштампованная заготовка. Образцы диаметром 8 мм и толщиной 0,45 мм деформировали при комнатной температуре на наковальне Бриджмана кручением на 5 оборотов при давлении 4 ГПа. После деформации образцы отжигали в интервале температур $300 \ldots 500^{\circ} \mathrm{C}$ в течение 5 минут. Оценивали среднюю площадь и объемную долю первичных и вторичных фаз, а также частиц $\mathrm{SiC}$ в исходном, деформированном и отоженном образцах. Проанализировали изменение легированности твердого раствора в исследуемых состояниях. Показано, что интенсивная пластическая деформация привела к дроблению присутствующих в сплаве частиц, средний размер которых уменьшался: кремния с 4,4 0,1

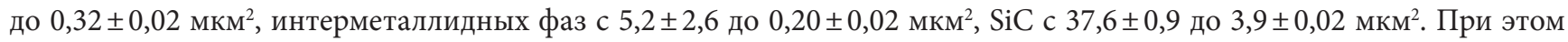
произошло частичное растворение избыточных фаз, что способствовало формированию пересыщенного твердого раствора. Последующий отжиг привел к распаду пересыщенного алюминиевого твердого раствора с выделением нетипичных для сплава вторичных фаз различной морфологии: глобулярного кремния, стержнеобразной фазы, обогащенной $\mathrm{Ni}, \mathrm{Si}$ и Fе и более компактной фазы, содержащей Si и Cu. При этом, чем выше была температура отжига, тем интенсивнее протекали процессы распада пересыщенного твердого раствора и тем больше была объемная доля частиц кремния и интерметаллидных фаз. С ростом температуры отжига увеличивалась и их средняя площадь. Изменения средней площади и объемной доли частиц $\mathrm{SiC}$ при отжигах деформированного сплава не наблюдались.

Ключевые слова: ИПД, алюминий, твердый раствор, распад. 


\section{1. Введение}

Методы интенсивной пластической деформации (ИПД) активно используются для формирования ультрамелкозернистой и нанокристаллической структур в алюминиевых сплавах и композициях на их основе, что приводит к существенному изменению их физических и механических свойств [1-3]. При этом, одновременно со структурным изменением матрицы сплавов, в процессе деформации может происходить изменение их фазового состава, которое обусловлено ускоренным распадом пересыщенного твердого раствора [4-7] и др., либо растворением частиц вторых фаз, в том числе и алюминидов переходных металлов (ПМ) [8-12].

При отжиге деформированных алюминиевых сплавов наряду с возвратом и рекристаллизацией, в термоупрочняемых сплавах могут протекать процессы распада твердого раствора.

В случае ИПД пересыщенные твердые растворы являются метастабильными [13] и при нагреве претерпевают ускоренный распад. В некоторых случаях, имеет место изменение кинетики распада, в результате чего могут быть сформированы структуры, отличающиеся от структур, полученных традиционной (т.е. при нормальных условиях) обработкой. Эти процессы подробно рассмотрены, например, в [4, 5 ,14-17].

Однако кроме ускоренного распада в алюминиевых сплавах при отжигах после ИПД были обнаружены фазы нетипичные для традиционной обработки. Например, авторы работы [15] показали, что ИПД, реализованное кручением под высоким давлением, и последующий отжиг при $350^{\circ} \mathrm{C}$ в течение 1 часа алюминиевого сплава 2024 (Al-Cu-Mg) привели к выделению из пересыщенного твердого раствора совокупности частиц вторичных фаз $\mathrm{Al}_{3} \mathrm{Fe}, \mathrm{Al}_{6} \mathrm{Mn}, \mathrm{Al}_{2} \mathrm{Cu}$ и $\mathrm{Al}_{2} \mathrm{CuMg}$, хотя при традиционной термообработке для сплавов системы Al-Cu-Mg типичными считаются $\mathrm{Al}_{2} \mathrm{Cu}$ и/или $\mathrm{Al}_{2} \mathrm{CuMg}$ [18].

Целью настоящей работы являлась оценка влияния ИПД, реализованной кручением под высоким давлением (КВД), и отжига на фазовый состав композиционного сплава АК12MМгH/18\%SiCp.

\section{2. Материал и методики исследования}

В качестве материала для исследований был выбран композиционный сплав, основой для которого служил алюминиевый литейный термоупрочныемый сплав АК12ММгН, армированный 18\% частиц SiC. Химический состав сплава соответствовал ГОСТ 1583-93: Al-13Si-1,5Cu-1,3Ni-1,3Mg-(0,7Fe-0,2Mn-0,2Cr-0,2Zn$0,2 \mathrm{Ti}-0,05 \mathrm{~Pb}-0,01 \mathrm{Sn})^{1}$. Композиционный сплав был получен жидкой штамповкой (ЖШ) [19].

Дисковые образцы диаметром 8 мм были вырезаны из исходной заготовки и утонены до толщины 0,45 мм. ИПД осуществляли кручением при комнатной температуре на наковальне Бриджмена под давлением 4 ГПа на 5 оборотов. При этом, согласно [2,3], логарифмическая степень деформации на периферии образца состав-

1 Состав сплава указан в вес.\%. Суммарная доля примесей не превышала 1,1 вес.\%. ляла $e=6,2$. Затем деформированные образцы отжигали в муфельной печи ЭКПС-50 при температурах 300, 400 и $500^{\circ} \mathrm{C}$ в течение 5 минут.

Структурный и фазовый анализ композиционного сплава исследовали в исходном, деформированном и отожженном состояниях. В деформированном и отожженных образцах исследования проводили на их периферии в кольцевой зоне диаметром 3 мм.

Энергодисперсионный (ЭДС/EDS) анализ твердого раствора сплава во всех состояниях, а также элементный состав частиц вторичных фаз проводили на РЭМ Tescan VEGA 3 LMH FEG, оснащенном приставкой фирмы «INCA Crystal». Для корректной идентификации вторичных фаз, деформированный образец подвергали отжигу в печи при $500^{\circ} \mathrm{C}$ в течение 2 часов, что способствовало увеличению их размера.

Статистическую обработку всех результатов количественной оценки проводили с доверительной вероятностью 95\%.

\section{3. Результаты исследований и их обсуждение}

Типичная исходная микроструктура композиционного сплава приведена на рис. 1а. Структура состояла из $\alpha$-Al твердого раствора, частиц избыточных фаз (эвтектического кремния $\{\mathrm{Si}\}$ и интерметаллидных фаз $\{\mathrm{IPh}\}: \mathrm{Mg}_{2} \mathrm{Si}$, $\left.\pi-\mathrm{Al}_{8} \mathrm{FeMg}_{3} \mathrm{Si}_{6}, \mathrm{Q}-\mathrm{Al}_{5} \mathrm{Cu}_{2} \mathrm{Mg}_{8} \mathrm{Si}, \mathrm{T}\left(\mathrm{Al}_{9}\right)-\mathrm{Al}_{9} \mathrm{FeNi}\right)$, а также достаточно равномерно распределенных армирующих включений SiC. Более подробный анализ фазового состава исследуемого сплава описан в ранней работе авторов [19]. Количественные характеристики структурных составляющих приведены в табл. 1.

Интенсивная пластическая деформация привела к существенному дроблению как избыточных фаз сплава, так и более твердых армирующих частиц $\mathrm{SiC}$ (рис. 1b) c уменьшением объемной доли интерметаллидных и кремниевых фаз (табл. 1).

Так как исследуемый сплав был получен жидкой штамповкой, в процессе которой из-за высокой скорости охлаждения происходило пресыщение твердого раствора легирующими элементами, а также формирование структуры с высокой плотностью дислокаций [20], предполагалось, как и в случае [6-10], протекание деформационного старения. Однако в нашем случае, по результатам ЭДС анализа, ИПД привела к увеличению содержания легирующих и примесных элементов в $\alpha$-Al, способствуя дополнительному пересыщению твердого

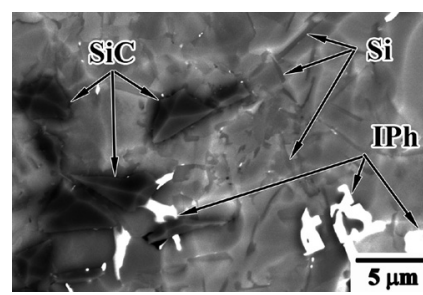

a

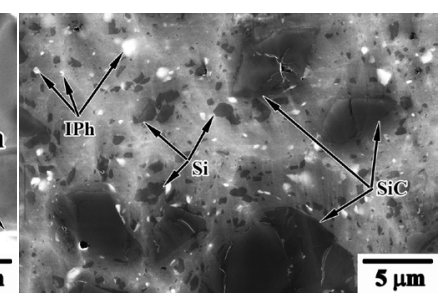

b
Рис. 1. Типичная структура сплава в исходном жидкоштампованном (a) и КВД (b) состояниях.

Fig. 1. The typical structure of alloy in the initial liquid forging (LF) (a) and HPT (b) states. 
Табл. 1. Средняя площадь (S) и объемная доля (V) частиц кремния (Si), первичных (Pr) и вторичных (Sec) интерметаллидных фаз (IPh), SiC в зависимости от режимов обработки.

Table 1. Average size (S) and the volume fraction (V) of the silicon (Si), primary (Pr) and secondary (Sec) intermetallic phases (IPh), SiC according to process conditions.

\begin{tabular}{|c|c|c|c|c|c|c|}
\hline $\begin{array}{l}\text { Фаза } \\
\text { Phase }\end{array}$ & & $\begin{array}{c}\text { ЖШ } \\
\text { LF }\end{array}$ & $\begin{array}{l}\text { КВД } \\
\text { НРТ }\end{array}$ & $300^{\circ} \mathrm{C}$ & $400^{\circ} \mathrm{C}$ & $500^{\circ} \mathrm{C}$ \\
\hline \multirow{2}{*}{$\mathrm{Si}$} & $\mathrm{S}, \mu \mathrm{m}^{2}$ & $4,4 \pm 0,1$ & $0,32 \pm 0,02$ & $0,37 \pm 0,02$ & $0,61 \pm 0,07$ & $0,97 \pm 0,04$ \\
\hline & $\mathrm{V}, \%$ & $17 \pm 1$ & $11 \pm 1$ & $11 \pm 1$ & $13 \pm 1$ & $17 \pm 1$ \\
\hline \multirow{3}{*}{$\mathrm{IPh}$} & $\mathrm{S}^{\mathrm{Pr}}, \mu \mathrm{m}^{2}$ & $5,2 \pm 2,6$ & \multicolumn{4}{|c|}{$0,20 \pm 0,02$} \\
\hline & $\mathrm{S}^{\mathrm{Sec}}, 10^{-3} \mu \mathrm{m}^{2}$ & - & - & $7,2 \pm 0,6$ & $19,4 \pm 1,3$ & $40,1 \pm 8,6$ \\
\hline & V, \% & $22 \pm 1$ & $4 \pm 1$ & $7 \pm 1$ & $8 \pm 1$ & $10 \pm 1$ \\
\hline \multirow{2}{*}{$\mathrm{SiC}$} & $\mathrm{S}, \mu \mathrm{m}^{2}$ & $37,6 \pm 0,9$ & \multicolumn{4}{|c|}{$3,9 \pm 0,2$} \\
\hline & $\mathrm{V}, \%$ & $17 \pm 1$ & \multicolumn{4}{|c|}{$16 \pm 1$} \\
\hline
\end{tabular}

раствора (рис. 2). Аналогичные результаты демонстрировали бинарные и тройные сплавы Al-ПМ $[9,10]$.

При последующем отжиге произошел ускоренный распад пересыщенного (являющегося результатом ИПД) твердого раствора с образованием частиц вторичных фаз (рис. 3а). Столь стремительные процессы зарождения и роста частиц при отжиге деформированного сплава обусловлены неравновесным его состоянием после ИПД. Следует отметить, что при отжиге в тех же температурно-временных условиях исходного жидкоштампованного композиционного материала частиц вторичных фаз обнаружено не было (рис. 3b).

Как известно [18], в зависимости от состава сплава системы Al-Mg-Si-Cu и режимов термической обработки могут формироваться следующие вторичные фазы: $\mathrm{Al}_{2} \mathrm{Cu}, \mathrm{Mg}_{2} \mathrm{Si}, \mathrm{Al}_{2} \mathrm{CuMg}$ и $\mathrm{Al}_{4} \mathrm{CuMg}_{5} \mathrm{Si}_{4}$.

Для подтверждения элементного состава вторичных фаз провели картирование по основным элементам (рис. 4). Максимальная плотность окраски на каждой карте показывала наличие тех или иных элементов в фазе. Затем, для идентификации вторичных фаз провели анализ спектров с областей, соответствующих наибольшей яркости элементов на картах (рис. 5). Численные значения содержания элементов накопленных спектров приведены в табл. 2 .

Согласно результатам ЭДС анализ, вторичные фазы не являются типичными для сплава АК12ММгН, поскольку, во-первых, при старении в сплавах системы $\mathrm{Al}-\mathrm{Mg}$-Si с содержанием магния 0,2 вес.\% и более кремний связывается в соединение $\mathrm{Mg}_{2} \mathrm{Si}$ [21]; во-вторых, обнаруженные вторичные фазы не соответствует ни одному из упомянутых ранее для сплава системы Al-Mg-Si-Cu.

Как показали результаты количественной оценки, с ростом температуры отжига средняя площадь и объемная доля частиц кремния возрастала (табл. 1). Это связано с тем, что в процессе распада пересыщенного твердого раствора, кроме зарождения и роста дисперсной вторичной фазы кремния, активно протекали процессы роста раздробленных при ИПД эвтектических частиц кремния. Наиболее интенсивно эти процессы протекали при температуре отжига 400 и $500^{\circ} \mathrm{C}$.
Средняя площадь первичных интерметаллидных фаз в процессе отжига не менялась. При этом с ростом температуры отжига средняя площадь вторичных фаз интерметаллидов увеличивалась одновременно с их объемной долей.

Количественные изменения средней площади и объемной доли частиц $\mathrm{SiC}$ в отожженном сплаве обнаружены не были.

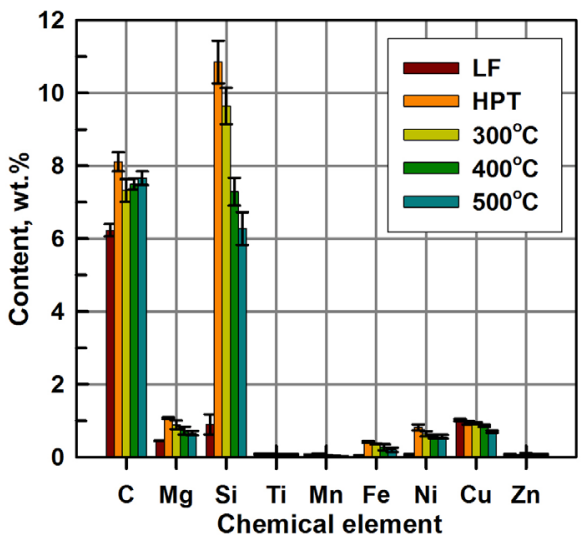

Рис. 2. Результаты ЭДС анализа алюминиевого твердого раствора в зависимости от режимов обработки (LF - жидкая штамповка, НРТ - кручение под высоким давлением).

Fig. 2. The results of EDS analysis of aluminum solid solution according to the process conditions (LF - liquid forging, HPT high pressure torsion).

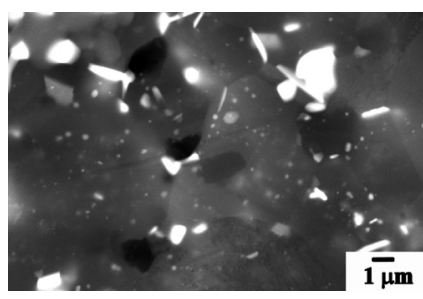

a

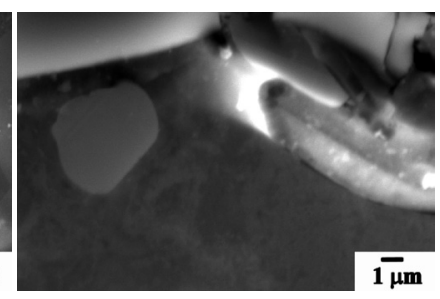

b
Рис. 3. Типичная структура сплава в деформированном (а) и исходном (b) состоянии после отжига при $500^{\circ} \mathrm{C}$ в течение 5 мин.

Fig. 3. The typical structure of alloy in the HPT (a) and initial liquid forging (b) states after annealing at $500^{\circ} \mathrm{C}$ for 5 minutes. 

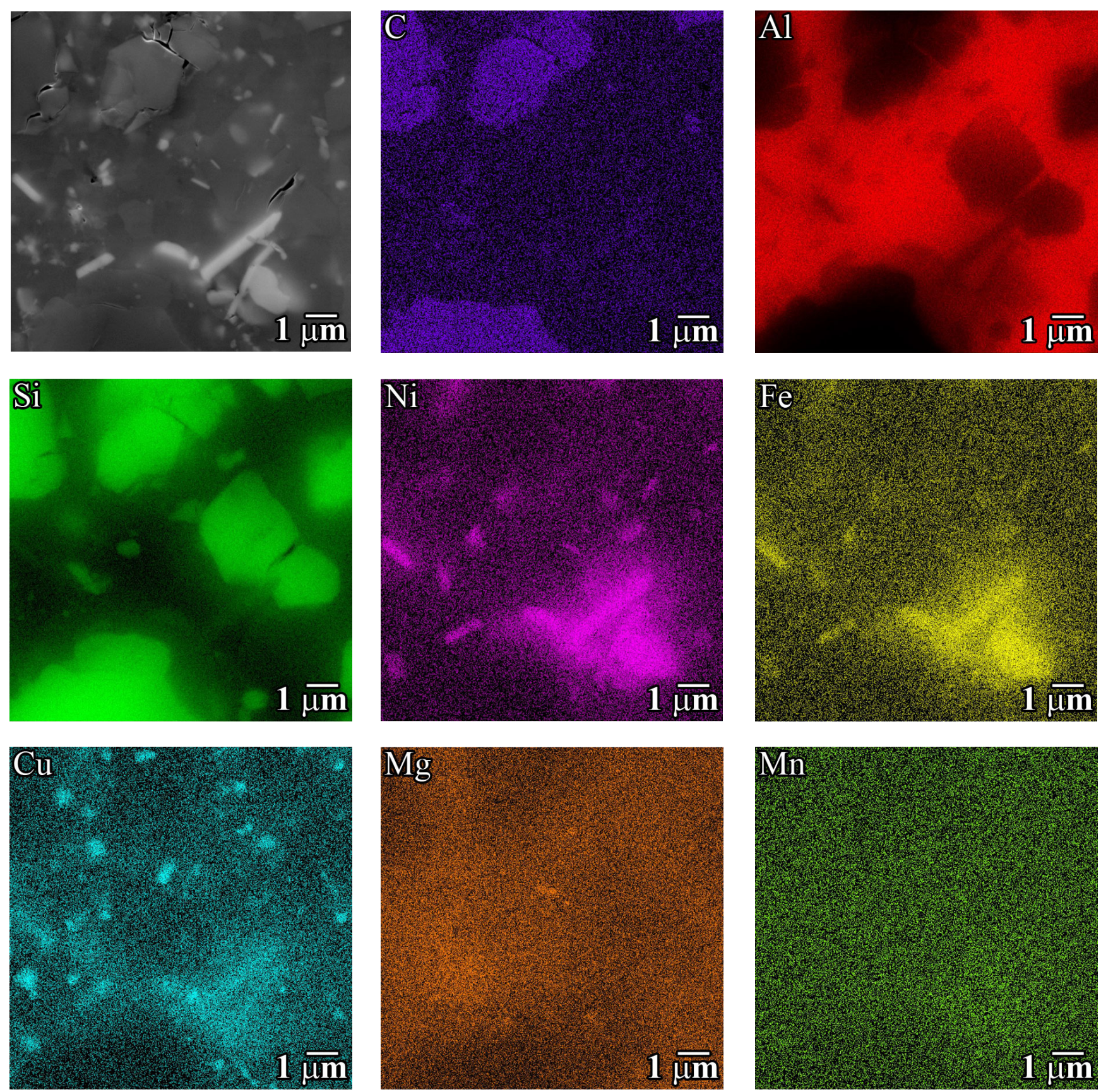

Pис. 4. (Color online) Изображение во вторичных электронах и картирование на этом участке по основным химическим элементам сплава после ИПД и отжига при $500^{\circ} \mathrm{C}$ в течение 2 часов.

Fig. 4. (Color online) The secondary electron image and the mapping to this region on the main elements of alloy after post-HPT annealing at $500^{\circ} \mathrm{C}$ for 2 hours.

Вышеописанные количественные изменения в структуре при отжиге деформированного сплава согласуются с результатами проведенного ЭДС анализа твердого раствора. При отжиге деформированных образцов содержание растворенных элементов в твердом растворе уменьшалось (рис. 2), и чем выше была температура отжига, тем интенсивнее протекали процессы его распада.

\section{4. Заключение}

В процессе ИПД при комнатной температуре композиционного сплава АК12MMгH-18\%SiCp, наряду с дроблением присутствующих частиц, имело место частичное растворение избыточных фаз с образованием аномально пересыщенного алюминиевого твердого раствора. При этом признаков растворения фазы $\mathrm{SiC}$ при деформации не наблюдалось. Последующий отжиг в течение 5 мин и в интервале температур $300 \ldots 500^{\circ} \mathrm{C}$ привел к распаду пересыщенного алюминиевого твердого раствора с образованием нетипичных для матричного сплава частиц вторичных фаз: глобулярного кремния, стержнеобразной фазы, обогащенной $\mathrm{Ni}, \mathrm{Si}$ и $\mathrm{Fe}$ и более компактной фазы, содержащей $\mathrm{Si}$ и $\mathrm{Cu}$. Кремниевая фаза активно зарождалась и росла при температурах выше $400^{\circ} \mathrm{C}$, в то время как интерметаллидные фазы - во всем исследуемом интервале температур. 


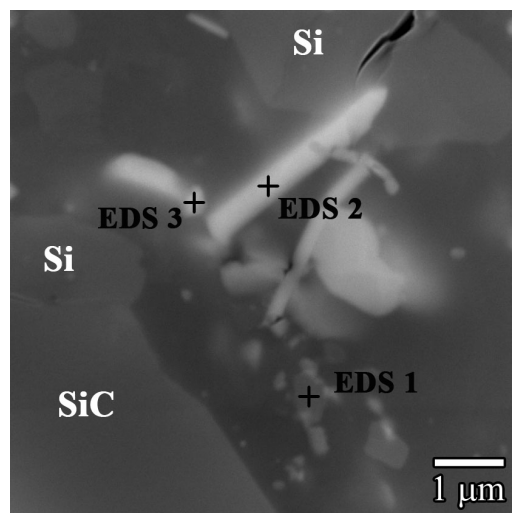

Рис. 5. Изображение во вторичных электронах с указанием мест накопления спектров при ЭДС анализе с частиц вторичных фаз.

Fig. 5. The secondary electron image and the spectrum accumulation points of secondary phases at the EDS analysis.

Табл. 2. Результаты ЭДС анализа частиц вторичных фаз. Table 2. The results of EDS analysis of of the secondary phases.

\begin{tabular}{|c|c|c|c|c|c|c|c|c|}
\hline & $\%$ & C & Al & Mg & Si & $\mathrm{Fe}$ & $\mathrm{Ni}$ & $\mathrm{Cu}$ \\
\hline \multirow{2}{*}{ 离 } & wt & 15,30 & 35,19 & 0,08 & 47,42 & 0,13 & 0,29 & 1,60 \\
\hline & at & 29,62 & 30,32 & 0,08 & 39,24 & 0,05 & 0,11 & 0,58 \\
\hline \multirow{2}{*}{ 次 } & wt & 9,40 & 70,58 & 0,16 & 5,88 & 2,80 & 7,99 & 3,19 \\
\hline & at & 20,33 & 67,92 & 0,18 & 5,43 & 1,30 & 3,53 & 1,30 \\
\hline \multirow{2}{*}{ 䓃 } & wt & 7,10 & 80,20 & 0,12 & 7,42 & 0,23 & 0,40 & 4,53 \\
\hline & at & 15,09 & 75,93 & 0,13 & 6,75 & 0,11 & 0,18 & 1,82 \\
\hline
\end{tabular}

\section{Литература/References}

1. Severe plastic deformation: toward bulk production of nanostructured materials/B.S. Altan ed. New York, Nova science publ., Inc. (2006) $612 \mathrm{p}$.

2. A. P. Zhilyaev, T. G. Langdon. Prog. Mat. Sci. 53, 893 - 979 (2008), Doi: 10.1016/j.pmatsci.2008.03.002

3. A. Azushima, R. Kopp, A. Korhonen, D. Y. Yang, F. Micari, G. D. Lahoti, P. Groche, J. Yanagimoto, N. Tsuji, A. Rosochowski, A. Yanagida. CIRP Annals Manufacturing Technology, 57, $716-735$ (2008), Doi:10.1016/j.cirp.2008.09.005

4. M. Murayama, Z. Horita, K. Hono. Acta mater. 49, 21 - 29 (2001), Doi:10.1016/S1359-6454 (00) 00308-6

5. S. Zang, W. Hu, R. Berghammer, G. Gottstein. Acta mater. 58, 6695-6705 (2010), Doi:10.1016/j. actamat.2010.08.034

6. A.A. Mazilkin, B. B. Straumal, E. Rabkin, B. Baretzky, S. Enders, S. G. Protasova, O. A. Kogtenkova, R.Z. Valiev. Acta Mater. 54, $3933-3939$ (2006), Doi:10.1016/j. actamat.2006.04.025

7. I.G. Brodova, I.G. Shirinkina, A.N. Petrova, O. V. Antonova, V.P. Pilugin. Phys. Met. Metallogr. 111(6), 659-667 (2011) (in Russian) [И.Г. Бродова,
И.Г. Ширинкина, А.Н. Петрова, О.В. Антонова, В.П. Пилюгин. Физика металлов и металловедение. 111(6), 659-667 (2011).]

8. O.N. Senkov, F.H. Froes, V.V. Stolyarov, R.Z. Valiev, J.Liu. Scripta Materialia, 38(10), 1511-1516, 1998, Doi:10.1016/S1359-6462 (98) 00073-6

9. Г.Р. Халикова, К.С. Швец, В.Г. Трифонов. Письма о Материалах, 5(2), 220-224 (2015) (in Russian) [G. R. Khalikova, K. S. Shvets, V. G. Trifonov. Letters on materials 5(2),. 220 - 224 (2015)], Doi: 10.22226/2410-3 535-2015-2-220-224

10. I.G. Brodova, D. V. Bashlikov, M.S. Nikitin, I. G. Shirinkina, T. I. Yablonskikh. Phys. Met. Metallogr. 98(1), 83-92 (2004) (in Russian) [И.Г. Бродова, Д.В. Башлыков, М.С. Никитин, И.Г. Ширинкина, Т.И. Яблонских. Физика металлов и металловедение. 98(1), 83 - 92 (2004).]

11. I.G. Brodova, I.G. Shirinkina, O.V. Antonova, A.V. Chirkova, S.V. Dobaykin. Maltseva. Russian metallurgy (Metally). 4, 25-32 (2009) (in Russian) [И.Г. Бродова, И.Г. Ширинкина, О.В. Антонова, А.В. Чиркова, С.В. Добаткин, В.В. Захаров. Деформация и разрушение материалов. 4, 25-32 (2009).]

12. K. Shvets, G. Khalikova, E. Korznikova, V. Trifonov. AIP Conference Proceedings, 1683, 020213 (2015), Doi: $10.1063 / 1.4932903$

13. V.V. Stolyarov, R.Z. Valiev. Nanomaterials by Sever Plastic Deformation. Proceedings of the Conference «Nanomaterials by Sever Plastic Deformation NANOSPD2». Vienna, Austria. 2002. p. 125 - 130.

14. Y. Huang, J.D. Robson, P.B. Prangnell. Acta Mater. 58, 1643 - 1657 (2010), Doi:10.1016/j.actamat.2009.11.008

15. G. Angella, P. Bassani, A. Tuissi, M. Vedani. Materials Transactions, 45(7), $2282-2287$ (2004), Doi:10.2320/m atertrans.45.2282

16. L. Zhen, W. D. Fei, S. B. Kang, H. W. Kim. Materials Science. 32, 1895 - 1902 (1997), Doi: 10.1023/A:1018569226499

17. A. Alhamidi, Z. Horita. Materials Science and Engineering A 622, 139 - 145 (2015), Doi: 10.1016/j.msea.2014.11.009

18. L.F. Mondolfo. Aluminum alloys: structure and properties. Butterworths. (1976) 971p.

19. G. R. Khalikova, R.V. Kal'shchikov, K.S. Shvets, V.G. Trifonov. Basic Problems of Material Science. 12(4), 458-465 (2015) (in Russian) [Г.Р. Халикова, Р.В. Кальщиков, К.С. Швец, В.Г. Трифонов. Фундаментальные проблемы современного материаловедения. 12(4), 458 - 465 (2015)]

20. T.N. Lipchin. Structure and properties of nonferrous alloys solidified under pressure, Moscow, Metallurgy (1994) 128p. (in Russian) [Т.Н. Липчин. Структура и свойства цветных сплавов, затвердевших под давлением, М., Металлургия. 1994. 128c.].

21. N.A. Belov. Phase composition of commercial and perspective aluminum alloys. Moscow, MISIS. (2010) 511 p. (in Russian) [Н. А. Белов. Фазовый состав промышленных и перспективных алюминиевых сплавов. М., МИСиС. 2010. 511с.] 